\title{
On the Equivalence of Scaling, Light-Cone Singularities and Asymptotic Behaviour of the Jost-Lehmann Spectral Function
}

\author{
E. Brüning and P. Stichel \\ Department of Theoretical Physics, University of Bielefeld, Bielefeld \\ Federal Republic of Germany
}

Received December 8, 1973

\begin{abstract}
For a local amplitude we prove a one-to-one correspondence between properly defined scaling, the leading light-cone singularity and the asymptotic behaviour of the corresponding Jost-Lehmann spectral function in the sense of distribution theory. The cases of canonical and non-canonical scaling are considered.
\end{abstract}

\section{Introduction}

The experimental discovery of Bjorken scaling in deep inelastic lepton-nucleon scattering at SLAC and DESY is nowadays part of the folklore in present elementary particle physics.

Most of the theoretical attempts to understand scaling phenomena start from an asymptotic expansion of causal commutators near the light cone [1]. But the authors of Ref. [1] only show, that an inverse power behaviour near the light cone of the form ${ }^{1}$

$$
\tilde{V}(x, p) \underset{x^{2} \rightarrow 0}{\simeq} \Theta\left(x^{2}\right)\left(x^{2}\right)^{\beta-2} f(x \cdot p)
$$

leads for $\beta>0$ to Bjorken scaling in momentum space with a power $v^{-\beta}$. The non-leading terms in the LC-expansion (1), which are in general not power-behaved, have been analysed by several other people [2-4] by means of the Riemann-Lebesgue theorem. The results of Ref. [2,3] show, that a one-to-one correspondence between scaling and the LC-limit demands for additional mathematical assumptions if scaling is understood as a convergence of a sequence of functions. This difficulty remains if one defines scaling as the regularization ${ }^{2}$ of the distribution $X(q, p)$ taken at infinity along a light-like direction [4]. Only for a restricted

\footnotetext{
${ }^{1}$ Our notation is the usual one [2]: For the process $\gamma_{v i r t}+N \rightarrow N+$ "anything" we denote by $q$ and $p$ the momenta of the virtual photon and nucleon respectively. We define the Bjorken (scaling) limit by $v \rightarrow \infty(v:=q \cdot p)$ for $q^{2} / v$ fixed. function.

${ }^{2}$ Regularization of a distribution is defined as its convolution product with a test
} 
class of distributions this difficulty has been overcome quite recently by taking into account locality [5].

Other attempts for explaining scaling start with the Jost-Lehmann (JL) or the Deser-Gilbert-Sudarshan integral representation for matrix elements of causal commutators by assuming from the beginning a nice asymptotic behaviour of the corresponding spectral function $[2,6-8]$. In this way the usually assumed light-cone dominance could be derived too.

Quite recently it has been shown by Zavialov [9], that an asymptotic power behaviour of the JL-spectral function is even necessary for the usual LC-behaviour, if both limits are understood in the sense of distribution theory. In this way Zavialov was the first, who derived rather rigorously scaling from a given LC-behaviour. But it has been an open problem up to now, whether the assumed asymptotic behaviour of spectral functions is a necessary condition for explaining scaling.

In this paper we will prove, that there is a one-to-one correspondence between scaling, light-cone dominance and asymptotic power behaviour of the JL-spectral function, if all these limits are understood as limits of sequences of distributions with respect to all variables.

The paper is organized as follows: In Section 2 we formulate scaling, LC-behaviour and JL-asymptotic in the sense of distribution theory. The equivalence of scaling and LC-behaviour will be shown in Section 3. Some additional comments on the equivalence of LC-behaviour and JLasymptotic are given in Section 4. Finally, we will draw some conclusions from the present results.

\section{Formulation of Scaling, LC-Behaviour and JL-Asymptotic}

The Fourier transform of the one-particle matrix element of the electromagnetic current commutator $W_{\mu \sigma}(q, p)$ is expressed in a Lorentz covariant and gauge invariant way in terms of two invariant amplitudes $V_{i}(q, p), i=1,2$ which are free of kinematical singularities and zeros [10]

$$
\begin{aligned}
& W_{\mu \sigma}(q, p) \\
& =\left\{q^{2} p_{\mu} p_{\sigma}-v\left(p_{\mu} q_{\sigma}+q_{\mu} p_{\sigma}\right)+g_{\mu \sigma} v^{2}\right\} V_{2}(q, p) \\
& \quad+\left\{q_{\mu} q_{\sigma}-q^{2} g_{\mu \sigma}\right\} V_{1}(q, p) .
\end{aligned}
$$

It can be shown, that both $V_{i}$ are Fourier transforms of local distributions $[7,11]$.

In the following we study an amplitude $V(q, p)$ as a representative for any of the two $V_{i}$. Let us start by listing the properties of $V(q, p)$, which follow from the usual axioms of quantum field theory [12]. 
Condition A. A distribution $V(q, p)$ defined for $p^{2}=1$ satisfies condition $\mathrm{A}$, if

$\left(\mathrm{A}_{1}\right) \quad V(\Lambda q, \Lambda p)=V(q, p) \quad \forall \Lambda \in L_{+}^{\uparrow} \quad$ Lorentz invariance

$\left(\mathrm{A}_{2}\right) \quad V(-q, p)=-V(q, p) \quad$ Crossing

$\left(\mathrm{A}_{3}\right) \quad V(q, p)=0$ for $q^{2}+2|v|<0 \quad$ Spectrum

$\left(\mathrm{A}_{4}\right)^{3} \tilde{V}(x, p)=0$ for $x^{2}<0, \forall p \quad$ Locality

$\left(\mathrm{A}_{5}\right)^{4} \quad V(q, p) \in \mathscr{S}^{\prime}\left(\mathbb{R}^{4}\right)$ for fixed $p \quad$ Temperedness.

Due to condition $\left(\mathrm{A}_{1}\right), V$ is a $O_{+}(3)$-invariant tempered distribution in the rest system $p=(1, \mathbf{0})$. We define

$$
V(q):=V(q,(1,0)) .
$$

Hence $V(q) \in \mathscr{S}^{\prime}\left(\mathbb{R}^{4}, O_{+}(3)\right)$.

Due to the topological isomorphism between $\mathscr{S}^{\prime}\left(\mathbb{R}^{3}, O_{+}(3)\right)$ and $\mathscr{S}^{\prime}\left(\mathbb{R}_{+}\right)$we may define uniquely a tempered distribution $V_{1}$ of the $O_{+}(3)$-invariants only:

$$
\left\langle V_{1}\left(q_{0}, \varrho\right), \phi f\left(q_{0}, \varrho\right)\right\rangle=\langle V(q), f(q)\rangle \forall f \in \mathscr{S}\left(\mathbb{R}^{4}\right)
$$

with

We have [13]

$$
\phi f\left(q_{0}, \varrho\right):=(4 \pi)^{-1} \int_{|\omega|=1} d \omega f\left(q_{0}, \sqrt{\varrho} \omega\right)
$$

$$
\phi f \in \mathscr{S}\left(\mathbb{R}^{1} \times \mathbb{R}_{+}^{1}\right)
$$

and therefore

$$
V_{1}\left(q_{0}, \varrho\right) \in \mathscr{S}^{\prime}\left(\mathbb{R}^{1} \times \mathbb{R}_{+}^{1}\right)
$$

There exists a unique extension of $V_{1}$ to a distribution $V_{2} \in \mathscr{S}^{\prime}\left(\mathbb{R}^{2}\right)_{-}{ }^{5}$ :

$$
\begin{aligned}
&\left\langle V_{2}\left(q_{0}, w\right), \varphi\left(q_{0}, w\right)\right\rangle=\left\langle V_{1}\left(q_{0}, \varrho\right), \frac{1}{\sqrt{\varrho}} \varphi\left(q_{0}, \sqrt{\varrho}\right)\right\rangle \\
& \forall \varphi \in \mathscr{S}\left(\mathbb{R}^{2}\right)_{-}
\end{aligned}
$$

and

$$
V_{2}\left(q_{0}, w\right)=0 \quad \text { on } \quad \mathscr{S}\left(\mathbb{R}^{2}\right) \backslash \mathscr{S}\left(\mathbb{R}^{2}\right)_{-} .
$$

${ }^{3}$ Here and in the following we denote by $\tilde{T}$ the Fourier transform of a distribution $T$.

4 In the usual axiomatic framework temperedness of matrix elements of field operators holds if these are taken with respect to proper (wave packet) states. For reasons of simplicity we restrict ourselves to improper (plane wave) states.

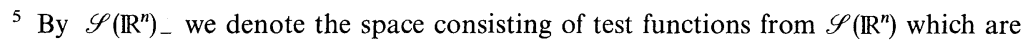
antisymmetric in all variables. 
Croșsing symmetry $\left(\mathrm{A}_{2}\right)$, which has already been used in (5), leads to the representation

with

$$
V_{2}\left(q_{0}, w\right)=V_{2}^{+}\left(q_{0}, w\right)-V_{2}^{+}\left(-q_{0}, w\right)
$$

$$
\text { supp } V_{2}^{+}=\operatorname{supp} V_{2} \cap\left\{\left(q_{0}, w\right) \mid q_{0} \geqq 0\right\} .
$$

From (6a) we conclude, that $V_{2}^{+}$is only unique up to a tempered distribution $T$ which is symmetric in $q_{0}$ and has support at the point $q_{0}=0$ only. Hence

$$
T\left(q_{0}, w\right)=\sum_{v=0}^{N} a_{v}(w) \delta^{(2 v)}\left(q_{0}\right) .
$$

For the following it is advantageous to define a tempered distribution $F(u, v)$ by means of the relation

$$
F\left(\frac{1}{2}\left(q_{0}+w+1\right), \frac{1}{2}\left(q_{0}-w+1\right)\right):=V_{2}^{+}\left(q_{0}, w\right) .
$$

From the antisymmetry of $V_{2}^{+}$with respect to $W$ we obtain the symmetry relation

$$
F(u, v)=-F(v, u) .
$$

The support of $F$ follows immediately from the definition of $F$ and the spectrum condition $\left(\mathrm{A}_{3}\right)$

$$
\begin{aligned}
\text { supp } F \cong\left\{(u, v) \mid u \geqq \frac{1}{2}, \frac{1}{4 u} \leqq v \leqq u\right\} \\
\cup\left\{(u, v) \mid v \geqq \frac{1}{2}, \frac{1}{4 v} \leqq u \leqq v\right\} .
\end{aligned}
$$
forms.

We are now prepared to state the scaling condition in two alternative

Definition 1 (Strong scaling).

$V(q)$ shows strong scaling of degree $\beta$, if there exists a real constant $\beta$, such that ${ }^{6}$

$$
\begin{gathered}
\lim _{\lambda \rightarrow+\infty} \lambda^{\beta-1} F(\lambda u, v) \exists=F_{\beta}(u, v) \text { in } \mathscr{S}^{\prime}\left(\mathbb{R}^{2}\right), \\
F_{\beta} \neq 0 \text { if } F \neq 0 .
\end{gathered}
$$

From Definition 1 and the support of $F$ we conclude, that $F_{\beta}(u, v)$ is a homogeneous distribution of degree $1-\beta$ on $(0, \infty)$ with respect to $u$, i.e. [13]

$$
\begin{gathered}
F_{\beta}(u, v)=u^{1-\beta} F_{\beta}(v) \\
\text { on } O_{+}^{2}, \quad \text { where } O_{+}^{2}:=\{(u, v) \mid u>0, v>0\} .
\end{gathered}
$$

This limit is just the naively expected result.

\footnotetext{
${ }^{6}$ This definition reduces the freedom in $(6 b)$ for $\beta>2$.
} 
It is well known, that strong scaling is violated for renormalizable interactions by logarithmic terms in finite order of perturbation theory. A formulation which is adapted to this situation has been given in the related case of the short-distance behaviour by Steinmann [14].

Lemma 1 (Weak scaling). There exists a real constant $\beta$, such that

(i) $\lim _{\lambda \rightarrow+\infty} \lambda^{\beta^{\prime}-1} F(\lambda u, v) \exists=0$ in $\mathscr{S}^{\prime}\left(\mathbb{R}^{2}\right) \forall \beta^{\prime}<\beta$,

(ii) $\nexists \lim _{\lambda \rightarrow+\infty} \lambda^{\beta^{\prime}-1} F(\lambda u, v)$ in $\mathscr{S}^{\prime}\left(\mathbb{R}^{2}\right), \beta^{\prime}>\beta$.

Such a behaviour of $F$ we call weak scaling of degree $\beta$.

For the proof of Lemma 1 we refer to the proof of Lemma 5.1 in Steinmann's paper [14].

Weak scaling of degree $\beta$ is a consequence of strong scaling of degree $\beta$, but not vice versa. In order to illustrate this by means of an example consider a distribution $F_{1}$ which satisfies relation (8) and shows strong scaling of degree $\beta$. Then we define a distribution $F_{2}$

$$
F_{2}(u, v):=\left(\ln ^{\alpha}\left(u+u_{0}\right)+\ln ^{\alpha}\left(v+u_{0}\right)\right) F_{1}(u, v)
$$

which shows weak scaling of degree $\beta$ but not strong scaling.

Our formulation of strong scaling is, in the case of pointwise convergent sequences of functions, equal to the usual form of Bjorken scaling with $\omega:=-q^{2} / 2 v \underset{v \rightarrow+\infty}{\longrightarrow} 1-2 v$ and $u \rightarrow v$.

Similarly we treat the leading LC-behaviour in the following.

We define a distribution $\tilde{V}^{1}$ of the $O_{+}(3)$-invariants $x_{0}$ and $\boldsymbol{x}^{2}$ by means of the chain of relations $\left(\forall f \in \mathscr{S}\left(\mathbb{R}^{4}\right)\right)$

$$
\begin{gathered}
\left\langle\tilde{V}^{1}\left(x_{0}, \sigma\right), \phi \tilde{f}\left(x_{0}, \sigma\right)\right\rangle=\left\langle\tilde{V}\left(x_{0}, \boldsymbol{x}\right), \tilde{f}\left(x_{0}, \boldsymbol{x}\right)\right\rangle \\
=(2 \pi)^{4}\left\langle V\left(q_{0}, \boldsymbol{q}\right), f\left(q_{0}, \boldsymbol{q}\right)\right\rangle=(2 \pi)^{4}\left\langle V_{1}\left(q_{0}, \varrho\right), \phi f\left(q_{0}, \varrho\right)\right\rangle .
\end{gathered}
$$

In exactly the same way as in case of $V_{1}\left(q_{0}, \varrho\right)$ we extend $\tilde{V}^{1}\left(x_{0}, \sigma\right)$ to a distribution $\tilde{V}^{2} \in \mathscr{S}^{\prime}\left(\mathbb{R}^{2}\right)_{\text {- }}$. By means of Eqs. (5), (6), and (12) we obtain

$$
\tilde{V}^{2}\left(x_{0}, x\right)=-4 \pi i \int d q_{0} \int d w \sin q_{0} x_{0} \cdot \sin w x V_{2}^{+}\left(q_{0}, w\right) .
$$

Due to crossing $\left(\mathrm{A}_{2}\right)$ and locality $\left(\mathrm{A}_{4}\right)$ we may define a distribution $\bar{V} \in\left(\mathscr{S}\left(\mathbb{R}_{+}^{1}\right) \otimes \mathscr{S}\left(\mathbb{R}^{1}\right)_{-}\right)^{\prime}$ by $^{7}$

$$
\tilde{V}^{2}\left(x_{0}, \varkappa\right)=\varepsilon\left(x_{0}\right) \bar{V}\left(x_{0}^{2}-\varkappa^{2}, \varkappa\right) .
$$

7 The formal relation (14) has to be understood as follows: For a given $\tilde{V}^{2}$ we obtain $\bar{V}$ by means of

$$
\langle\bar{V}(\eta, x), g(\eta, x)\rangle=\left\langle\tilde{V}^{2}\left(x_{0}, \varkappa\right), x_{0} g\left(x_{0}^{2}-\varkappa^{2}, x\right)\right\rangle \forall g \in \mathscr{S}\left(\mathbb{R}_{+}^{1} \times \mathbb{R}^{1}\right)
$$

and for a given $\bar{V}$ we obtain $\tilde{V}^{2}$ by means of

$$
\begin{aligned}
\left\langle\tilde{V}^{2}\left(x_{0}, x\right), f\left(x_{0}, x\right)\right\rangle= & \left\langle\bar{V}(\eta, x), \frac{1}{2}\left(\eta+x^{2}\right)^{-1 / 2}\left[f\left(\sqrt{\eta+\varkappa^{2}}, x\right)\right.\right. \\
& \left.\left.-f\left(-\sqrt{\eta+x^{2}}, x\right)\right]\right\rangle \forall f \in \mathscr{S}\left(\mathbb{R}^{2}\right) .
\end{aligned}
$$


Two alternative forms of leading LC-behaviour are defined in terms of $\bar{V}$ now.

Definition 2 (Strong LC-behaviour) ${ }^{8}$.

$\bar{V}(\eta, x)$ shows strong LC-behaviour of degree $\gamma$, if there exists a real constant $\gamma$, such that

$$
\begin{aligned}
\lim _{\lambda \rightarrow+\infty} \lambda^{\gamma-2} \bar{V}(\eta / \lambda, \chi) \exists & =\bar{V}_{\gamma}(\eta, \chi) \text { in }\left(\mathscr{S}\left(\mathbb{R}_{+}^{1}\right) \otimes \mathscr{S}\left(\mathbb{R}^{1}\right)_{-}\right)^{\prime} \\
\bar{V}_{\gamma} & \neq 0 \text { if } \bar{V} \neq 0 .
\end{aligned}
$$

Definition 2 implies, that $\bar{V}_{\gamma}(\eta, \varkappa)$ is a homogeneous distribution of degree $\gamma-2$ on $[0, \infty)$ with respect to $\eta$, i.e. [13]

$$
\bar{V}_{\gamma}(\eta, \chi)=\frac{(\eta)_{+}^{\gamma-2}}{\Gamma(\gamma-1)} g_{\gamma}(\chi) .
$$

This result is equal to the usually assumed form of the leading LCsingularity.

With the same arguments as in the case of scaling we introduce another formulation which is adapted to the situation in perturbation theory.

Lemma 2 (Weak LC-behaviour). There exists a real constant $\gamma$, such that

$\left.\begin{array}{l}\text { (i) } \lim _{\lambda \rightarrow+\infty} \lambda^{\gamma^{\prime}-2} \bar{V}(\eta / \lambda, x) \exists=0 \\ \text { (ii) } \nexists \lim _{\lambda \rightarrow+\infty} \lambda^{\gamma^{\prime}-2} \bar{V}(\eta / \lambda, x)\end{array}\right\} \begin{array}{ll}\forall \gamma^{\prime}<\gamma \\ \text { in } \begin{array}{l}\left(\mathscr{S}\left(\mathbb{R}_{+}^{1}\right) \otimes \mathscr{S}\left(\mathbb{R}^{1}\right)_{-}\right)^{\prime} . \\ \gamma^{\prime}>\gamma\end{array}\end{array}$

Such a behaviour of $\bar{V}$ we call weak LC-behaviour of degree $\gamma$.

The proof of Lemma 2 is just the Fourier analogue of the proof of Lemma 1.

Again weak LC-behaviour of degree $\gamma$ is a consequence of strong LC-behaviour of degree $\gamma$.

As an immediate consequence of condition A the $O_{+}(3)$-invariant tempered distribution $V(q)$ satisfies a JL-representation.

Lemma 3 (Jost-Lehmann [15]). A distribution $V(q)$ satisfies conditions $\left(\mathrm{A}_{2}\right)-\left(\mathrm{A}_{5}\right)$, if and only if there exists a $\mathrm{O}_{+}(3)$-invariant tempered distribution $\Psi(s, \boldsymbol{u})$ with

such that

$$
\operatorname{supp} \Psi \subseteq\left\{(s, \boldsymbol{u})|| \boldsymbol{u} \mid \leqq 1, s \geqq s_{0}(\boldsymbol{u})=\left(1-\sqrt{1-\boldsymbol{u}^{2}}\right)^{2}\right\}
$$

$$
\langle V(q), f(q)\rangle=\langle\Psi(s, \boldsymbol{u}), T f(s, \boldsymbol{u})\rangle \forall f \in \mathscr{S}\left(\mathbb{R}^{4}\right)
$$

with

$$
T f(s, \boldsymbol{u}):=\int d^{4} q f(q) \varepsilon\left(q_{0}\right) \delta\left(q_{0}^{2}-(\boldsymbol{q}-\boldsymbol{u})^{2}-s\right) .
$$

\footnotetext{
${ }^{8}$ Compare Ref. [9].
} 
By means of the Fourier transform of $\Psi$ with respect to $\boldsymbol{u}$ we introduce a distribution $\tilde{\Psi}_{1} \in \mathscr{S}^{\prime}\left(\mathbb{R}_{+}^{2}\right)$ of the $O_{+}(3)$-invariants only

$$
\left\langle\tilde{\Psi}_{1}(s, \sigma), \phi \tilde{f}(s, \sigma)\right\rangle=(2 \pi)^{3}\langle\Psi(s, \boldsymbol{u}), f(s, \boldsymbol{u})\rangle
$$

together with its odd extension $\tilde{\Psi}_{2}(s, \varkappa)$

$$
\begin{gathered}
\left\langle\tilde{\Psi}_{2}(s, \varkappa), g(s, \varkappa)\right\rangle=\left\langle\tilde{\Psi}_{1}(s, \sigma), \sigma^{-1 / 2} g\left(s, \sigma^{1 / 2}\right)\right\rangle \\
\forall g \in \mathscr{S}\left(\mathbb{R}_{+}^{1}\right) \otimes \mathscr{S}\left(\mathbb{R}^{1}\right) .
\end{gathered}
$$

With that and the definition of $\bar{V}$ we obtain as an alternative form of the JL-representation

$$
\langle\bar{V}(\eta, x), g(\eta, x)\rangle=\left\langle\tilde{\Psi}_{2}(s, x), \varphi_{g}(s, x)\right\rangle
$$

where the mapping

$$
g(\eta, \chi) \mapsto \varphi_{g}(s, \chi):=i(2 \pi)^{2} \int_{0}^{\infty} d \eta J_{0}(\sqrt{\eta s}) \frac{\partial}{\partial \eta} g(\eta, x)
$$

is a topological isomorphism of $\mathscr{S}\left(\mathbb{R}_{+}^{1}\right) \times \mathscr{S}\left(\mathbb{R}^{1}\right)_{-}$.

In terms of $\tilde{\Psi}_{2}$ we may formulate the JL-asymptotic now.

Definition 3 (Strong JL-asymptotic) ${ }^{9}$.

$\tilde{\Psi}_{2}(s, x)$ shows strong JL-asymptotic of degree $\delta$, if there exists a real constant $\delta$, such that

$$
\begin{gathered}
\lim _{\lambda \rightarrow+\infty} \lambda^{\delta} \tilde{\Psi}_{2}(\lambda s, \chi) \exists=\tilde{\Psi}_{\delta}(s, x) \quad \text { in }\left(\mathscr{S}\left(\mathbb{R}_{+}^{1}\right) \otimes \mathscr{S}\left(\mathbb{R}^{1}\right)_{-}\right)^{\prime} \\
\tilde{\Psi}_{\delta} \neq 0, \text { if } \quad \tilde{\Psi}_{2} \neq 0 .
\end{gathered}
$$

Definition 3 implies that $\tilde{\Psi}_{\delta}(s, \varkappa)$ is a homogeneous distribution of degree $-\delta$ on $[0, \infty)$ with respect to $s$, i.e. [13]

$$
\tilde{\Psi}_{\delta}(s, \varkappa)=\frac{(s)_{+}^{-\delta}}{\Gamma(1-\delta)} \varphi_{\delta}(\varkappa) .
$$

Again a weak form of JL-asymptotic is obtained from the following lemma.

Lemma 4 (Weak JL-asymptotic). There exists a real constant $\delta$, such that
(i) $\lim _{\lambda \rightarrow+\infty} \lambda^{\delta^{\prime}} \tilde{\Psi}_{2}(\lambda s, \varkappa) \exists=0$
$\forall \delta^{\prime}<\delta$
(ii) $\nexists \lim _{\lambda \rightarrow+\infty} \lambda^{\delta^{\prime}} \tilde{\Psi}_{2}(\lambda s, x)$ in $\left(\mathscr{S}\left(\mathbb{R}_{+}^{1}\right) \otimes \mathscr{S}\left(\mathbb{R}^{1}\right)_{-}\right)^{\prime}$.

Such a behaviour we call weak JL-asymptotic of degree $\delta$.

\footnotetext{
${ }^{9}$ Compare Ref. [9].
} 
The proof of Lemma 4 is the same as that of Lemma 1.

Now we give a general representation formula for a distribution $\bar{V}$ satisfying strong LC-behaviour.

Lemma 5. $A$ distribution $\bar{V}(\eta, x)$ which satisfies condition $A$ shows strong LC-behaviour of degree $\gamma$ if and only if there exists a natural number $n$ with $n+\gamma-2 \geqq 0$, such that

$$
\bar{V}(\eta, x)=D_{\eta}^{n} \eta^{n+\gamma-2} \bar{V}^{0}(\eta, x)
$$

where $\bar{V}^{0}(\eta, x)$ exhibits the following properties:

(i) it is continuous and polynomially bounded in $(\eta, x) \in \mathbb{R}_{+}^{1} \times \mathbb{R}^{1}$,

(ii) it is an odd entire function of exponential Type 1 in $x \in \mathbb{C}, \forall \eta \in \mathbb{R}_{+}^{1}$, $\eta$ fixed.

By means of Lebesgue's bounded convergence criterium it is easy to show, that the representation Eq. (22) is sufficient for strong LCbehaviour of degree $\gamma$. The proof that (22) is even necessary for strong LC-behaviour will be given in Appendix A, just as the proof of the following.

Corollary. Sufficient for the validity of (22) is strong LC-behaviour of degree $\gamma$ of $\bar{V}(\eta, x)$ on $\mathbb{R}_{\varepsilon}^{1}, \forall \varepsilon>0$,

$$
\mathbb{R}_{\varepsilon}^{1}:=\{\varkappa|| \varkappa \mid>\varepsilon>0\} .
$$

\section{Equivalence of Scaling and Leading LC-Behaviour}

The equivalence of scaling and leading LC-behaviour is stated in our following main Theorem 1 by means of Definitions 1 and 2 given in Section 2.

Theorem 1. For a distribution $V(q)$, which satisfies condition $A$, we have

(a) Strong scaling of degree $\beta$ implies strong $L C$-behaviour of degree $\beta \forall \beta \in \mathbb{R}^{1}$.

(b) Strong LC-behaviour of degree $\beta>0$ implies strong scaling of degree $\beta$.

First we derive statement (a) of Theorem 1. We have to ask, whether

$$
I_{\lambda}:=\lambda^{\beta-2}\langle\bar{V}(\eta / \lambda, x), g(\eta, x)\rangle
$$

has a finite limit for $\lambda \rightarrow+\infty$ for every $g \in \mathscr{S}\left(\mathbb{R}_{+}^{1}\right) \otimes \mathscr{S}\left(\mathbb{R}^{1}\right)_{-}$supposed strong scaling of degree $\beta$ holds.

By means of Eqs. (14), (13), and (7) we obtain ${ }^{10}$

$$
I_{\lambda}=8 \pi i \lambda^{\beta-1}\left\langle e^{i x_{0}} \tilde{F}\left(x_{0}-\varkappa, x_{0}+\varkappa\right), x_{0} g\left(\left(x_{0}^{2}-\varkappa^{2}\right) \lambda, \varkappa\right)\right\rangle .
$$

10 By reasons of symmetry the non-unique part $T$ of $F$ gives no contribution to (24). 
If we introduce in (24) the new variables

$$
\eta_{-}=\lambda\left(x_{0}-x\right), \quad \eta_{+}=x_{0}+x
$$

we obtain

with

$$
I_{\lambda}=2 \pi i \lambda^{\beta-2}\left\langle\tilde{F}\left(\frac{\eta_{-}}{\lambda}, \eta_{+}\right), \varphi_{\lambda}\left(\eta_{-}, \eta_{+}\right)\right\rangle
$$

$$
\varphi_{\lambda}\left(\eta_{-}, \eta_{+}\right):=\left(\eta_{+}+\frac{\eta_{-}}{\lambda}\right) e^{\frac{i}{2}\left(\eta_{+}+\frac{\eta_{-}}{\lambda}\right)} g\left(\eta_{-} \cdot \eta_{+}, \frac{1}{2}\left(\eta_{+}-\frac{\eta_{-}}{\lambda}\right)\right) .
$$

In Appendix B we show, that $\left\{\varphi_{\lambda}\left(\eta_{-}, \eta_{+}\right)\right\}_{\lambda \rightarrow+\infty}$ converges in the topology of $\mathscr{S}\left(\mathbb{R}^{2}\right), \forall g \in \mathscr{S}\left(\mathbb{R}_{+}^{1}\right) \otimes \mathscr{S}\left(\mathbb{R}_{\varepsilon}^{1}\right), \forall \varepsilon>0$. As strong scaling of degree $\beta$ is equivalent to

$$
\lim _{\lambda \rightarrow+\infty} \lambda^{\beta-2} \tilde{F}\left(\frac{\eta_{-}}{\lambda}, \eta_{+}\right) \exists \quad \text { in } \quad \mathscr{S}^{\prime}\left(\mathbb{R}^{2}\right) .
$$

$\lim _{\lambda \rightarrow+\infty} I_{\lambda}$ exists due to a convergence criterium of distribution theory [16] $\forall g \in \mathscr{S}\left(\mathbb{R}_{+}^{1}\right) \otimes \mathscr{S}\left(\mathbb{R}_{\varepsilon}^{1}\right), \forall \varepsilon>0$. By means of the corollary to Lemma 5 we conclude that $\bar{V}(\eta, \chi)$ satisfies the representation Eq. (22). Hence, due to Lemma $5, \bar{V}(\eta, \chi)$ shows strong LC-behaviour of degree $\beta$.

In order to derive statement (b) of Theorem 1 we start with the quantity

$$
J_{\lambda}:=\lambda^{\beta-1}\langle F(\lambda u, v), g(u, v)\rangle, \quad \beta>0
$$

and ask for the existence of $\lim _{\lambda \rightarrow+\infty} J_{\lambda} \forall g \in \mathscr{S}\left(\mathbb{R}^{2}\right)$ supposed strong LCbehaviour of degree $\beta$ holds. By means of Eqs. (7) and (13) we obtain for $J_{\lambda}$

$$
J_{\lambda}=-i(2 \pi)^{-1} \lambda^{\beta-2}\left\langle\bar{V}(\eta / \lambda, x), h_{\lambda}(\eta, x)\right\rangle
$$

with

$h_{\lambda}(\eta, x):=\left(\eta / \lambda+x^{2}\right)^{-1 / 2}$

$\cdot \operatorname{Re}\left\{e^{i x} f\left(\lambda\left(\sqrt{\frac{\eta}{\lambda}+x^{2}}-x\right), \sqrt{ }+x\right)-e^{-i x} f(\lambda(\sqrt{ }+x), \sqrt{ }-x)\right\}$

where

$$
f(a, b):=e^{-i b} \tilde{g}(a, b) .
$$

According to Lemma 5, $\bar{V}$ satisfies the representation Eq. (22). Therefore, $J_{\lambda}$ is given by the integral

$$
J_{\lambda}=-i(2 \pi)^{-1}(-1)^{n} \int_{0}^{\infty} d \eta \int_{-\infty}^{\infty} d x \cdot x \cdot \hat{\bar{V}}^{0}\left(\frac{\eta}{\lambda}, x^{2}\right) \eta^{\beta+n-2}\left(\frac{\partial}{\partial \eta}\right)^{n} h_{\lambda}(\eta, x)
$$


where $\hat{\bar{V}}^{0}(a, b)$ is a polynomially bounded continuous function for $(a, b) \in \mathbb{R}_{+}^{2}$.

In Appendix $\mathrm{C}$ we show for the integrand in Eq. (30):

(i) it may be majorized for $\beta>0$ by a $\lambda$-independent absolute integrable function on $\mathbb{R}_{+}^{1} \times \mathbb{R}^{1}$.

(ii) it converges for $\lambda \rightarrow+\infty$ almost everywhere on $\mathbb{R}_{+}^{1} \times \mathbb{R}^{1}$.

Therefore, Lebesgue's bounded convergence criteria may be used for the evaluation of $\lim _{\lambda \rightarrow+\infty} J_{\lambda}$ leading to

$$
\lim _{\lambda \rightarrow+\infty} J_{\lambda} \exists=J_{\infty}
$$

with

$$
\begin{gathered}
J_{\infty}=\frac{-i}{\pi}(-1)^{n} P \int_{0}^{\infty} d x \int_{0}^{\infty} d \eta \hat{\bar{V}}^{0}\left(0, \varkappa^{2}\right)(\eta)_{+}^{\beta+n-2}\left(\frac{\partial}{\partial \eta}\right)^{n} \\
\cdot \operatorname{Re}\left[e^{-i x} \tilde{g}\left(\frac{\eta}{2 \varkappa}, 2 x\right)\right] .
\end{gathered}
$$

With this result we have proved strong scaling starting with strong LC-behaviour. In addition we may derive some interesting properties of the distributions $F_{\beta}(v)$ and $g_{\beta}(x)$ which occur in the asymptotic forms of $F$ and $\bar{V}$ [Eqs. (10), (15)] respectively.

Lemma 6. The distribution $g_{\beta}(\chi)$ defined by means of Eq. $(1,5)$ is an odd entire function of exponential Type 1 which is polynomially bounded for $\operatorname{Im} \varkappa=0$.

Lemma 6 is an immediate consequence of the representation Eq. (22) for $\bar{V}$.

Lemma $7^{11}$. The distribution $F_{\beta}(v)[E q .(10)]$ and $g_{\beta}(\chi)$ are related to each other according to $(\beta>0)$

$$
F_{\beta}(v)=-i \pi^{-1} \int_{0}^{\infty} d x g_{\beta}(x) x^{-1}(2 x)^{\beta-1} \cos \left[(2 v-1) x+\frac{\pi}{2}(\beta-1)\right] \text {. }
$$

For the proof of (32) we observe, that

$$
\tilde{g}\left(\frac{\eta}{2 \varkappa}, 2 \varkappa\right) \in \mathscr{S}\left(\mathbb{R}_{+}^{1},{ }_{\eta}\right), \quad \varkappa \neq 0 .
$$

Therefore, with Eqs. (31), (22), and (15) we obtain

$$
J_{\infty}=\frac{-i}{\pi} P \int_{0}^{\infty} d \varkappa g_{\beta}(\varkappa) x^{-1}\left\langle\frac{(\eta)_{+}^{\beta-2}}{\Gamma(\beta-1)}, \operatorname{Re}\left[e^{-i \varkappa} \tilde{g}\left(\frac{\eta}{2 \varkappa}, 2 \varkappa\right)\right]\right\rangle .
$$

\footnotetext{
11 Compare Gatto, Menotti Ref. [1] and - Ref. [17].
} 
Eq. (33) has to be compared with the asymptotic form of Eq. (26), which due tọ Eq. (10) takes the form

$$
J_{\infty}=\left\langle u^{1-\beta} F_{\beta}(v), g(u, v)\right\rangle .
$$

By means of well-known results on the Fourier cosine and sine transforms of $(\eta)_{+}^{\beta-2}$ the supposition (32) follows.

From the spectrum condition we know supp $F_{\beta} C(0, \infty)$. On the other hand the r.h.s. of Eq. (32) is symmetric (anti-symmetric) under the substitution $v \rightarrow 1-v$, if $\beta$ is equal to an odd (even) integer. Therefore, we obtain from Eq. (32) the well-known fact, that the scaling function $F_{\beta}$ has bounded support for integer $\beta=n$

$$
\text { supp } F_{n} \subset(0,1) \text {. }
$$

Our Theorem 1 may be extended immediately to the cases of weak scaling and weak LC-behaviour respectively.

Corollary 1. For a distribution $V(q)$, which satisfies condition $A$, we have:

(a) Weak scaling of degree $\beta \geqq 0$ implies weak LC-behaviour of degree $\beta$.

(b) Weak LC-behaviour of degree $\beta>0$ implies weak scaling of degree $\beta$.

For the proof of statement (a) we start with weak scaling of degree $\beta \geqq 0$. The weak scaling condition (i) in Lemma 1 implies via Theorem $1 \mathrm{a}$ the weak LC-condition (i) in Lemma 2. Now suppose we would have

$$
\lim _{\lambda \rightarrow+\infty} \lambda^{\beta^{\prime}-2} \bar{V}(\eta / \lambda, \chi) \exists \quad \text { in } \quad\left(\mathscr{S}\left(\mathbb{R}_{+}^{1}\right) \otimes \mathscr{S}\left(\mathbb{R}^{1}\right)_{-}\right)^{\prime} \quad \text { for } \quad \beta^{\prime}>\beta
$$

then, due to Theorem $1 \mathrm{~b}$, we conclude

$$
\lim _{\lambda \rightarrow+\infty} \lambda^{\beta^{\prime}-1} F(\lambda u, v) \exists \quad \text { in } \mathscr{S}^{\prime}\left(\mathbb{R}^{2}\right) \text { for } \quad \beta^{\prime}>\beta
$$

in contradiction to our assumption of weak scaling [Lemma 1, (ii)]. Therefore the weak LC-condition (ii), Lemma 2, is fulfilled.

In a similar fashion we may prove statement (b).

\section{On the Equivalence of Leading LC-Behaviour and JL-Asymptotic}

The equivalence of strong LC-behaviour and strong JL-asymptotic of degree $\beta$ respectively has already been shown very recently in Ref. [9] by similar methods. For reasons of completeness and further reference we repeat the result. 
Theorem 2 (Zavialov [9]). Suppose $V(q)$ satisfies condition A. Then $\bar{V}\left(x^{2}, x\right)$ shows strong $L C$-behaviour of degree $\beta$ if and only if $\tilde{\Psi}_{2}(s, x)$ [defined by Lemma 3 and Eq. (18)] shows strong JL-asymptotic of degree $\beta$, $\forall \beta \in \mathbb{R}^{1}$.

For the proof of Theorem 2 (compare [9]) we use the JL-representation in the form of Eq. (19) and obtain

$$
\lambda^{\beta-2}\left\langle\bar{V}\left(\frac{\eta}{\lambda}, x\right), g(\eta, x)\right\rangle=\lambda^{\beta}\left\langle\tilde{\Psi}_{2}(\lambda s, x), \varphi_{g}(s, x)\right\rangle
$$

where - due to Eq. (20) - the mapping $g \mapsto \varphi_{g}$ is a topological isomorphism of $\mathscr{S}\left(\mathbb{R}_{+}^{1}\right) \otimes \mathscr{S}\left(\mathbb{R}^{1}\right)_{-}$. Thus the stated result follows.

With the same arguments as given in Section 3 for Corollary 1 we may extend immediately Theorem 2 to the cases of weak LC-behaviour and weak JL-asymptotic respectively.

Corollary 2. Suppose $V(q)$ satisfies condition $A$. Then $\bar{V}\left(x^{2}, x\right)$ shows weak LC-behaviour of degree $\beta$ if and only if $\tilde{\Psi}_{2}(s, x)$ shows weak $J L$ asymptotic of degree $\beta, \forall \beta \in \mathbb{R}^{1}$.

Furthermore, we obtain from Eq. (35) proportionality between the functions $\varphi_{\beta}(x)$ and $g_{\beta}(\varkappa)$, which occur in the asymptotic forms of $\tilde{\Psi}_{2}$ and $\bar{V}$ respectively [9]:

$$
g_{\beta}(x)=-i(2 \pi)^{2} 2^{2(1-\beta)} \varphi_{\beta}(x) .
$$

Therefore, according to Lemma $6, \varphi_{\beta}(\varkappa)$ is an odd entire function which is polynomially bounded for $\operatorname{Im} x=0$ (this statement can be obtained from the JL-representation directly).

\section{Conclusions}

The combination of Theorems 1 and 2 leads to the supposed equivalence between scaling, leading LC-behaviour and JL-asymptotic in a region $\beta>0$ which contains the physical relevant interval $1 \leqq \beta \leqq 2$. Compared with Zavialov's work [9], we were able to derive leading LC-behaviour from scaling. In addition we extended the proof of equivalence to the cases of weak scaling, weak LC-behaviour and weak JL-asymptotic respectively.

Furthermore we conclude

(i) Singularities in the interior of the light cone don't contribute to the scaling limit [9].

In order to show that we define in agreement with Ref. [9]

$$
\bar{V}_{\chi}(\eta, \chi)=\chi(\eta) \bar{V}(\eta, x)
$$


with $\chi \in \mathscr{C}^{\infty}\left(\mathbb{R}_{+}^{1}\right)$,

$$
\chi(\eta) \equiv 1 \text { for } \eta>b>a>0, \quad \chi(\eta) \equiv 0 \text { for } 0 \leqq \eta<a .
$$

As can be shown easily

$$
\lambda^{\alpha} \chi\left(\frac{\eta}{\lambda}\right) g(\eta) \underset{\lambda \rightarrow+\infty}{\longrightarrow} 0
$$

in the topology of $\mathscr{S}\left(\mathbb{R}_{+}^{1}\right), \forall g \in \mathscr{S}\left(\mathbb{R}_{+}^{1}\right)$, and every real number $\alpha$.

Therefore,

in

$$
\lim _{\lambda \rightarrow+\infty} \lambda^{\gamma-2} \bar{V}_{\chi}\left(\frac{\eta}{\lambda}, x\right) \exists=0
$$

$$
\left(\mathscr{S}\left(\mathbb{R}_{+}^{1}\right) \otimes \mathscr{S}\left(\mathbb{R}^{1}\right)_{-}\right)^{\prime} \quad \forall \gamma \text { real },
$$

i.e. we say $\bar{V}_{\chi}$ shows strong scaling of degree $\infty$. Theorem $1 \mathrm{~b}$ implies, that the corresponding distribution $F_{\chi}(u, v)$ shows strong scaling of degree $\infty$. The same result has been obtained in Ref. [18] for special distributions $\bar{V}$ having singularities of the type $\delta^{(k)}\left(x^{2}-a\right), a>0$, only. In particular the authors of Ref. [18] pointed out the difference in the results for the scaling limit in the cases where $V(q)$ has been considered as a distribution or a point function.

(ii) Even for integer values of $\beta$ the leading $L C$-contribution, i.e. $g_{\beta}(x)$, is in general not determined from measured values of the scaling function $F_{\beta}(v)$ in the physical region $q^{2} \leqq 0$ alone (compare Ref. [4]): consider in agreement with Lemma 6 a polynomial Ansatz for $g_{n}(\varkappa)$, then the corresponding $F_{n}(v)$ is a distribution with point support at $v=1 / 2$.

(iii) The connection between leading LC-behaviour and equal-time commutation, supposed by many authors ${ }^{12}$, can be put on a rigorous mathematical basis.

Let us consider the most important example, the so-called "Schwinger term sum rule". By means of the representation Eq. (22) and Lebesgue's bounded convergence criteria one easily proves the following lemma: then

Lemma 8. Suppose $\bar{V}\left(x^{2}, x\right)$ shows strong LC-behaviour of degree 1 ,

$$
\lim _{\lambda \rightarrow+\infty} \lambda \frac{\partial}{\partial x_{0}} \tilde{V}\left(\frac{x_{0}}{\lambda}, \boldsymbol{x}\right) \exists=\left.\frac{g_{1}(x)}{x}\right|_{x=0} \delta(x) \text { in } \mathscr{S}^{\prime}\left(\mathbb{R}^{4}\right) \text {. }
$$

Inverting Eq. (32) we obtain the desired sum rule

$$
\left.\frac{g_{1}(x)}{x}\right|_{\varkappa=0}=-2 i \int_{0}^{1} d v F_{1}(v)
$$

12 Compare the first two papers of Ref. [1], Ref. [2, 4] and the second paper of Ref. [8]. 
where the integral on the r.h.s. has to be understood as a regularized one if necessary.

By the same method other equal-time sum rules, including their generalization for $\beta \neq 1$ may be derived.

Acknowledgement. We are very grateful to B.I. Zavialov for communicating his results prior to publication.

We acknowledge some important remarks by H. J. Borchers.

\section{Appendix A}

In this appendix we give a proof of Lemma 5 and the following corollary.

In order to show, that the representation Eq. (22) is necessary for strong LC-behaviour of degree $\gamma$ we proceed in four steps:

1. Denote by $\hat{V}(\eta, u)$ the Fourier transform of $\bar{V}$ with respect to $x$. From Lemma 3 we infer

$$
\text { supp } \hat{V} \subseteq\{(\eta, u) \mid \eta \geqq 0,-1 \leqq u \leqq 1\}, \hat{V}(\eta, u)=-\hat{V}(\eta,-u) .
$$

Strong LC-behaviour of degree $\gamma$ is therefore equivalent to the statement

$$
\begin{aligned}
\lim _{\lambda \rightarrow+\infty} \lambda^{\gamma-2} \hat{V}\left(\frac{\eta}{\lambda}, u\right) \exists & =\hat{V}_{\gamma}(\eta, u) \quad \text { in }\left(\mathscr{S}\left(\mathbb{R}_{+}^{1}\right) \otimes \mathscr{E}\left(\mathbb{R}^{1}\right)_{-}\right)^{\prime} \\
\hat{V}_{\gamma} & \neq 0 \text { if } \hat{V} \neq 0
\end{aligned}
$$

where $\hat{V}_{\gamma}$ exhibits the properties given by (A 1$)$.

2. As a tempered distribution $\hat{V}(\eta, u)$ is of finite order [16], $\exists p \in \mathbb{N}$ such that convergence of $\lambda^{\gamma-2} \hat{V}(\eta / \lambda, u)$ in $\left(\mathscr{S}\left(\mathbb{R}_{+}^{1}\right) \otimes \mathscr{E}\left(\mathbb{R}^{1}\right)_{-}\right)^{\prime}$ means convergence in $\left(\mathscr{S}_{p}\left(\mathbb{R}_{+}^{1}\right) \otimes \mathscr{E}\left(\mathbb{R}^{1}\right)_{-}\right)^{\prime}[16]$ where

$$
\mathscr{S}_{p}\left(\mathbb{R}_{+}^{1}\right):=\left\{\varphi \in \mathscr{C}^{p}\left(\mathbb{R}_{+}^{1}\right) \mid\|\varphi\|_{p}:=\sup _{\substack{\eta \geqq 0 \\ q \leqq p}}\left\{(1+\eta)^{p}\left|D^{q} \varphi(\eta)\right|\right\}<\infty\right\} .
$$

3. For a fixed $n$ with $n \geqq p+1$ and $n+\gamma-2 \geqq 0$ we define

with

$$
\bar{V}^{1}\left(\eta^{\prime}, x\right):=\left\langle\hat{V}(\eta, u), \Theta_{n-1}\left(\eta-\eta^{\prime}\right) \otimes \sin \varkappa u\right\rangle
$$

$$
\Theta_{n-1}(x):=\frac{x^{n-1}}{\Gamma(n)} \cdot \Theta(x) .
$$

Because of the support properties of $\hat{V}\left(\eta^{\prime}, u\right)$ and $\Theta_{n-1}\left(\eta-\eta^{\prime}\right)$ with respect to $\eta^{\prime}, \Theta_{n-1}\left(\eta-\eta^{\prime}\right)$ may be modified to give a test function in $\mathscr{S}_{p}\left(\mathbb{R}_{+}^{1}\right)$ without changing the 1.h.s. in (A 2) [19]. 
From that and the preceding results we conclude:

(i) $\bar{V}^{1}$ is continuous and polynomially bounded in

$$
(\eta, x) \in \mathbb{R}_{+}^{1} \times \mathbb{R}^{1},
$$

(ii) $\bar{V}^{1}$ is an odd entire function of exponential Type 1 in

$$
\varkappa, \forall \eta \in \mathbb{R}_{+}^{1}, \eta \text { fixed [20], }
$$

(iii) $D_{\eta}^{n} \bar{V}^{1}(\eta, \varkappa)=\bar{V}(\eta, \varkappa)$ in $\mathscr{S}^{\prime}\left(\mathbb{R}_{+, \eta}^{1}\right)$

$\forall x \in \mathbb{R}^{1}, x$ fixed,

(iv) $\lim _{\lambda \rightarrow+\infty} \lambda^{n+\gamma-2} \bar{V}^{1}\left(\frac{\eta}{\lambda}, x\right) \exists=\bar{V}_{\gamma}^{1}(\eta, x)$

$$
\forall x \in \mathbb{R}^{1}, x \text { fixed }
$$

where $\bar{V}_{\gamma}^{1}(\eta, \varkappa)=\left\langle\hat{V}_{\gamma}\left(\eta^{\prime}, u\right), \Theta_{n-1}\left(\eta-\eta^{\prime}\right) \otimes \sin \varkappa u\right\rangle$ exhibits the properties (i) and (ii).

4. From the preceding relations we conclude, that the function

$$
\bar{V}^{0}(\eta, x):=\eta^{-n-\gamma+2} \bar{V}^{1}(\eta, x)
$$

exhibits the supposed properties (i) and (ii) given in Lemma 5. We obtain finally

$$
\bar{V}(\eta, \varkappa)=D_{\eta}^{n} \eta^{n+\gamma-2} \bar{V}^{0}(\eta, \varkappa) .
$$

For the proof of the corollary we proceed again in four steps:

1. Due to the preceding results strong LC-behaviour of degree $\gamma$ on $\mathbb{R}_{\varepsilon}^{1}$ is equivalent to ${ }^{13}$ :

$$
\exists p, l \in \mathbb{N}: \bar{V}^{0}(\eta, \chi) \underset{\eta \rightarrow 0}{\longrightarrow} \bar{V}^{0}(\varkappa) \text { in } \mathscr{S}_{p, l}^{\prime}\left(\mathbb{R}_{\varepsilon}^{1}\right)
$$

i.e. [21] for the $p$-th primitive $\bar{V}_{0}^{0}(\eta, \chi)$ of $\bar{V}^{0}(\eta, \chi)$ we have

and

$$
\sup _{x \in \mathbb{R}_{\varepsilon}^{1}}\left(1+\varkappa^{2}\right)^{-l}\left|\bar{V}_{0}^{0}(\eta, x)-\bar{V}_{0}^{0}(x)\right| \underset{\eta \rightarrow 0}{\longrightarrow} 0
$$

$$
\bar{V}^{0}(\varkappa)=D^{p} \bar{V}_{0}^{0}(\varkappa) \quad \text { in } \quad \mathscr{S}_{p, l}^{\prime}\left(\mathbb{R}_{\varepsilon}^{1}\right) .
$$

In particular we conclude from (A 7)

$$
\left|\bar{V}_{0}^{0}(\eta, x)\right| \leqq K\left(1+\chi^{2}\right)^{l} \forall x \in \mathbb{R}_{\varepsilon}^{1} \forall \eta \in\left(0, \eta_{0}\right]
$$

with $K=K(\varepsilon, l, p)$.

2. We infer from (A 1), (A 2), (A 5), and (A 6) that $\bar{V}_{0}^{0}(\eta, \chi)$ is an odd entire function of exponential Type 1 in $x \forall \eta \in\left(0, \eta_{0}\right]$, $\eta$ fixed.

\footnotetext{
${ }^{13} \mathscr{S}_{p, l}\left(\mathbb{R}_{\varepsilon}^{1}\right):=\left\{\varphi \in \mathscr{C}^{p}\left(\mathbb{R}_{\varepsilon}^{1}\right) \mid\|\varphi\|_{p, l}:=\sup _{\mathbb{I R}_{\varepsilon}^{1}}\left[\left(1+\varkappa^{2}\right)^{l}\left|D^{p} \varphi(\chi)\right|\right]<\infty\right\}$.
} 
Define

$$
\bar{V}_{1}^{0}(\eta, x):=\left(\frac{\sin \delta x}{\delta x}\right)^{2 l} \bar{V}_{0}^{0}(\eta, x)
$$

with

$$
0<\delta<\frac{\pi-1}{2 l}
$$

$\bar{V}_{1}^{0}$ is an entire function of exponential Type $1+2 l \delta<\pi$.

According to (A 9) and (A 10) we have

$$
\left|\bar{V}_{1}^{0}(\eta, \varkappa)\right| \leqq K_{1}, \forall \eta \in\left(0, \eta_{0}\right], \forall \varkappa \in \mathbb{R}_{\varepsilon}^{1}, K_{1}=K_{1}(K, \delta) .
$$

For a sufficiently small $\varepsilon>0$ and the choice

$$
x_{n}=\frac{1}{2}+n, \quad n=0, \pm 1, \pm 2, \pm \cdots
$$

the suppositions of Theorem 10.5.3 in Ref. [22] are fulfilled ${ }^{14}$. Hence $\exists M<\infty$ with $M$ independent of $\eta$, such that

$$
\left|\bar{V}_{1}^{0}(\eta, x)\right| \leqq M K_{1}, \forall \eta \in\left(0, \eta_{0}\right], \forall x \in \mathbb{R}^{1} .
$$

With the choice of $\varepsilon \in\left(0, \frac{\pi}{2 \delta}\right)$ we have

$$
\left|\frac{\sin \delta x}{\delta x}\right| \geqq \frac{2}{\pi}, \quad \forall x \in[-\varepsilon, \varepsilon]
$$

and therefore due to (A 10) and (A 12)

$$
\left|\bar{V}_{0}^{0}(\eta, x)\right| \leqq\left(\frac{\pi}{2}\right)^{2 l} M K_{1}, \forall \eta \in\left(0, \eta_{0}\right], \forall \varkappa \in[-\varepsilon, \varepsilon] .
$$

Finally we obtain due to (A 7), (A 9), and (A 13)

and

$$
\left|\bar{V}_{0}^{0}(\eta, \varkappa)\right| \leqq K_{2} \cdot\left(1+\varkappa^{2}\right)^{l}, \forall \eta \in\left(0, \eta_{0}\right], \forall \varkappa \in \mathbb{R}^{1}
$$

with

$$
\bar{V}_{0}^{0}(\eta, x) \underset{\eta \rightarrow 0}{\Rightarrow} \bar{V}_{0}^{0}(x) i I \mathbb{R}_{\varepsilon}^{1}
$$

$$
K_{2}=K_{2}(p, l, \varepsilon, \delta)<\infty \text {. }
$$

3. Let $G \subset \mathbb{C}_{\varkappa}$ be a sufficiently large domain of compact closure with $G \cap \mathbb{R}_{\varepsilon}^{1} \neq \emptyset$. As $\bar{V}_{0}^{0}(\eta, x)$ is an entire function of exponential Type 1 for all $\eta \in\left(0, \eta_{0}\right]$, (A 14a) tells us that the restriction of $\left\{\bar{V}_{0}^{0}(\eta, x)\right\}_{\eta \in\left(0, \eta_{0}\right]}$ on $G$ are a uniformly bounded family of holomorphic functions, which according to (A 14b) converges uniformly for $\eta \rightarrow 0$ on each compact set $I \subset \mathbb{R}_{\varepsilon}^{1} \cap G \subset G$. Hence (Theorem $51 \mathrm{a}$ (Vitali) in Ref. [23])

$$
\bar{V}_{0}^{0}(\eta, \varkappa) \underset{\eta \rightarrow 0}{\Rightarrow} \bar{V}_{0}^{0}(\varkappa) i I G
$$

thus $\bar{V}_{0}^{0}(\varkappa)$ and - due to (A 8) $-\bar{V}^{0}(\varkappa)$ are holomorphic on $G$.

\footnotetext{
${ }^{14}$ We are grateful to H. J. Borchers to the hint at this theorem.
} 
4. As $G$ can be chosen arbitrarily large, $\bar{V}^{0}(x)$ is an odd entire function and - due to the preceding results - of exponential Type 1.

\section{Appendix B}

In this appendix we want to show, that the sequence

$$
\varphi_{\lambda}\left(\eta_{-}, \eta_{+}\right)=\left(\eta_{+}+\eta_{-} / \lambda\right) e^{\frac{i}{2}\left(\eta_{+}+\frac{\eta_{-}}{\lambda}\right)} g\left(\eta_{-} \cdot \eta_{+}, \frac{1}{2}\left(\eta_{+}-\frac{\eta_{-}}{\lambda}\right)\right)
$$

converges for $\lambda \rightarrow+\infty$ in the topology of

$$
\mathscr{S}\left(\mathbb{R}^{2}\right) \forall g(\eta, \varkappa) \in \mathscr{S}\left(\mathbb{R}_{+}^{1}\right) \otimes \mathscr{S}\left(\mathbb{R}_{\varepsilon}^{1}\right)_{-} \forall \varepsilon>0 .
$$

Proof. From (B 1) we conclude

(i) $\varphi_{\lambda} \in \mathscr{C}^{\infty}\left(\mathbb{R}^{2}\right) \forall \lambda \geqq 1$.

(ii) On each compact set $K \subset \mathbb{R}^{2} \varphi_{\lambda}$ converges uniformly together with all its derivatives to

$$
\varphi_{\infty}\left(\eta_{-}, \eta_{+}\right)=\eta_{+} e^{\frac{i}{2} \eta_{+}} g\left(\eta_{-} \cdot \eta_{+}, \frac{1}{2} \eta_{+}\right)
$$

(iii) The Schwartz-norms of $\varphi_{\lambda}$

$$
\left\|\varphi_{\lambda}\right\|_{n, m}=\sup _{\substack{|\mu| \leqq m \\(\eta+, \eta-) \in \mathbb{R}^{2}}}\left\{\left(1+\eta_{+}^{2}+\eta_{-}^{2}\right)^{\frac{n}{2}}\left|D^{\mu} \varphi_{\lambda}\left(\eta_{-}, \eta_{+}\right)\right|\right\}
$$

possess $\lambda$-independent upper bounds for $\lambda \geqq 1$,

$$
g \in \mathscr{S}\left(\mathbb{R}^{2}\right), \text { suppg } \leqq\{(\eta, x)|\eta \geqq 0,| x \mid \geqq \varepsilon>0\} .
$$

In order to show that, it suffices to derive $\lambda$-independent upper bounds for

$$
N_{l, m}^{(i, j)}(\lambda)=\sup _{\mathbb{R}^{2}}\left|\eta_{+}^{m} \eta_{-}^{l} g^{(i, j)}\left(\eta_{+}, \eta_{-}\right) \frac{1}{2}\left(\eta_{+}-\frac{\eta_{-}}{\lambda}\right)\right| .
$$

Combining properties (i)-(iii), we obtain, due to a well-known convergence criterium for sequences of test functions [13], the desired result.

\section{Appendix C}

In this appendix we want to show, that for the evaluation of the integral $\lim _{\lambda \rightarrow+\infty} J_{\lambda}$ defined by Eq. (30) the limit may be interchanged with the integration, i.e. Lebesgue's bounded convergence criterium is applicable. 
After we have performed the differentiations in the integrand, we introduce the substitution

$$
x=\lambda\left(\sqrt{\eta / \lambda+x^{2}}-x\right), \quad y=\sqrt{\eta / \lambda+x^{2}}+x
$$

and obtain

with

$$
J_{\lambda}=-i \frac{(-1)^{n}}{2 \pi} \sum_{l=0}^{n} \sum_{p=0}^{l} b_{l}^{(n)} \cdot\left(\begin{array}{c}
l \\
l-p
\end{array}\right) I_{l, p}^{(n)}(\lambda)
$$

$$
\begin{aligned}
I_{l, p}^{(n)}(\lambda):= & \int_{-\infty}^{\infty} d x \int_{-\infty}^{\infty} d y \hat{\bar{V}}^{0}\left(\frac{x \cdot y}{\lambda}, \frac{1}{4}\left(y-\frac{x}{\lambda}\right)^{2}\right) \\
& \cdot(x \cdot y)^{\beta-2+n} \lambda^{p-n} \frac{1}{2}\left(y-\frac{x}{\lambda}\right)\left[\frac{1}{2}\left(y+\frac{x}{\lambda}\right)\right]^{-2 n+l} \\
& \cdot \operatorname{Re}\left[e^{\frac{i}{2}\left(y-\frac{x}{\lambda}\right)} f^{(p, l-p)}(x, y)-e^{-\frac{i}{2}\left(y-\frac{x}{\lambda}\right)} f^{(p, l-p)}\left(\lambda y, \frac{x}{\lambda}\right)\right]
\end{aligned}
$$

where $f \in \mathscr{S}\left(\mathbb{R}^{2}\right), \beta+n-2 \geqq 0$ and $\hat{V}^{0}$ being continuous and polynomially bounded in both variables.

For the expression in the square bracket in (C2) we may write

$$
\begin{aligned}
& -\int_{0}^{y-\frac{x}{\lambda}} d \varrho \frac{\partial}{\partial \varrho}\left\{e^{\frac{i}{2}\left(y-\frac{x}{\lambda}-2 \varrho\right)} f^{(p, l-p)}(x+\lambda \varrho, y-\varrho)\right. \\
= & -\int_{0}^{y-\frac{x}{\lambda}} d \varrho e^{\frac{i}{2}\left(y-\frac{x}{\lambda}-2 \varrho\right)}\left\{-i f^{(p, l-p)}(x+\lambda \varrho, y-\varrho)\right. \\
& \left.-f^{(p, l-p+1)}(x+\lambda \varrho, y-\varrho)+\lambda f^{(p+1, l-p)}(x+\lambda \varrho, y-\varrho)\right\} .
\end{aligned}
$$

For the following it suffices to consider the most singular term of (C3) in connection with (C2), i.e.

$$
\begin{aligned}
& \hat{I}_{l, p}^{(n)}(\lambda):=\int_{-\infty}^{\infty} d x \int_{-\infty}^{\infty} d y \hat{\bar{V}}^{0}\left(\frac{x \cdot y}{\lambda}, \frac{1}{4}\left(y-\frac{x}{\lambda}\right)^{2}\right)(x \cdot y)^{\beta-2+n} \lambda^{p-n+1} \\
& \frac{\frac{1}{2}\left(y-\frac{x}{\lambda}\right)}{\left[\frac{1}{2}\left(y+\frac{x}{\lambda}\right)\right]^{2 n-l}} \operatorname{Re} \int_{0}^{y-\frac{x}{\lambda}} d \varrho e^{\frac{i}{2}\left(y-\frac{x}{\lambda}-2 \varrho\right)} f^{(p+1, l-p)}(x+\lambda \varrho, y-\varrho) .
\end{aligned}
$$

Now we have to distinguish three cases:

a) $n=0$ (and therefore $\beta \geqq 2$ ).

In that case the term proportional to $f(x, y)$ in the integrand of (C2) has for $\lambda \geqq 1$ the $\lambda$-independent absolutely integrable upper bound

$$
c_{0}|x \cdot y|^{\beta-2}(1+|x \cdot y|)^{n_{1}}\left[1+\frac{1}{2}|y+x|\right]^{m_{1}}|f(x, y)| .
$$


After the substitution $\lambda y \rightarrow x, \frac{x}{\lambda} \rightarrow y$ we obtain a similar estimate for the 2 nd term in (C2). Furthermore, for $y \neq 0$ the integrand in $(\mathrm{C} 2)$ converges to

$$
\hat{\bar{V}}^{0}\left(0, \frac{1}{4} y^{2}\right) \frac{1}{2} y(x \cdot y)^{\beta-2} \operatorname{Re}\left\{e^{-\frac{i}{2} y} f(x, y)\right\} .
$$

b) $1 \leqq l \leqq n$.

The term proportional to $f^{(p, l-p)}(x, y)$ in the integrand of $(\mathrm{C} 2)$ has for $\lambda \geqq 1$ and $\beta>0$ the $\lambda$-independent absolutely integrable upper bound

$$
c_{1}(1+|x \cdot y|)^{n_{2}}\left[1+\frac{1}{2}|x+y|\right]^{m_{2}}|x|^{l-1}|x \cdot y|^{\beta-1}\left|f^{(p, l-p)}(x, y)\right| .
$$

The second term in (C2) may be treated similarly. Again the pointwise convergence of the integrand is obvious for $y \neq 0$.

c) $l=0, n \geqq 1$.

In that case we consider the integrand in (C4). We have the $\lambda$ independent estimate

$$
\begin{aligned}
& \text { integrand }(\mathrm{C} 4) \mid \leqq C_{3}^{\prime}(1+|x \cdot y|)^{n_{3}}\left[1+\frac{1}{2}|y+x|\right]^{m_{3}} \\
& \cdot|x \cdot y|^{\beta-1}\left(\frac{|y|-\frac{|x|}{\lambda}}{|y|+\frac{|x|}{\lambda}}\right)^{2} \sup _{\varrho}\left|\Theta\left(\left|y-\frac{x}{\lambda}\right|-|\varrho|\right) f^{(1,0)}(x+\lambda \varrho, y-\varrho)\right| \\
& \leqq C_{3}(1+|x \cdot y|)^{n_{3}}\left[1+\frac{1}{2}|y+x|\right]^{m_{3}}|x \cdot y|^{\beta-1}\left[1+\frac{1}{2}|x+y|+|x \cdot y|\right]^{-N}
\end{aligned}
$$

with $N \in \mathbb{N}$ arbitrary. By means of a convenient choice of $N$ the expression on the r.h.s. of (C5) is absolutely integrable on $\mathbb{R}^{2}$ for $\beta>0$.

Again the pointwise convergence of the integrand is obvious for $y \neq 0$.

\section{References}

1. Jackiw, R., Van Royen, R., West, G. B.: Phys. Rev. D 2, 2473 (1970)

Leutwyler, H., Stern, J.: Nucl. Phys. B 20, 77 (1970)

Brandt, R., Preparata, G.: Nucl. Phys. B 27, 541 (1971)

Gatto, R., Menotti,P.: Phys. Rev. D 5, 1493 (1972)

Frishman, Y.: Schladming Lectures, 1971, Acta Phys. Austr. 34, 351 (1971)

2. Stichel,P.: Lectures given at the «Ecole Internationale de la Physique des Particules Elementaires», Basko Polje-Makarska (Yougoslavie), September 1971

3. Chen, T.W.: J. Math. Phys. 14, 287 (1972)

4. Leutwyler, H., Otterson,P.: Talk presented at the Frascati Meeting on "Outlook for Broken Conformal Symmetry", May 1972

5. Magg, M.: International Symposium on Electron and Photon Interactions at High Energies, Bonn, August 1973, Contribution Nr. 149

6. Brown,L.S.: Lectures delivered at the Boulder summer school, 1969

7. Bogolubov, N. N., Tavkhelidze, A.N., Vladimirov, V.S.: TM $\phi$ 12, 305 (1972) 
8. Brandt, R. A.: Phys. Rev. D 1, 2808 (1970)

Cornwall, J.M., Corrigan,D., Norton, R.E.: Phys. Rev. D3, 536 (1971)

Bhaumik, D., Greenberg,O.W., Mohapatra, R. N.: Phys. Rev. D6, 2989 (1972)

Vinciarelli,P., Weisz,P.: Phys. Rev. D 7, 3091 (1973)

9. Zavialov, B.I.: TM $\phi$ 17, 178 (1973)

10. Bardeen, W.A., Tung, W. K.: Phys. Rev. 173, 1423 (1968)

11. Meyer,J.W., Suura,H.: Phys. Rev. 160, 1366 (1967)

12. Streater,R.F., Wightman,A.S.: Die Prinzipien der Quantenfeldtheorie, BI-Hochschultaschenbücher, Bd. 435/435 a

13. Gelfand,I.M., Schilov, G.E.: Verallgemeinerte Funktionen (Distributionen), Vol. I. Berlin: VEB Deutscher Verlag der Wissenschaften, 1967

14. Steinmann, O.: Lecture Notes in Physics, Vol. 11, Berlin-Heidelberg-New York: Springer 1971

15. Jost, R., Lehmann, H.: Nuovo Cimento 5, 1598 (1957)

Dyson, F.J.: Phys. Rev. 110, 1460 (1958)

Wightman, A.S.: In: deWitt, C., Omnes, R. (Eds.): Relations de dispersion et particules élementaires. Paris: Hermann 1960

16. Gelfand, I.M., Schilov, G.E.: Verallgemeinerte Funktionen (Distributionen), Vol. II. Berlin: VEB Deutscher Verlag der Wissenschaften 1967

17. Orzalesi,C.A.: Phys. Rev. D7, 488 (1973)

18. Bhaumik,D., Greenberg,O.W.: Phys. Rev. D7, 3136 (1973)

19. Bros, J., Epstein, H., Glaser, V.: Commun. math. Phys. 6, 77 (1967)

20. Bremermann, H.: Distributions, complex variables, and Fourier transforms. Section 8.33. New York: Addison-Verlag Publ. Co. 1965

21. Wloka,J.: Lecture Notes in Mathematics, Vol. 82. Berlin-Heidelberg-New York: Springer 1969

Friedman, A.: Generalized functions and partial differential equations. Inc. Englewood Cliffs, N.J.: Prentice-Hall 1963

22. Boas, R.P., Jr.: Entire functions. New York: Academic Press Inc., Publishers 1954

23. Behncke,H., Sommer,F.: Theorie der analytischen Funktionen einer komplexen Veränderlichen. Berlin-Heidelberg-New York: Springer 1965

Communicated by R. Haag P. Stichel

E. Brüning

Universität Bielefeld

Fakultät für Physik

Abteilung Theoretische Physik

D-4800 Bielefeld

Herforder Straße 28

Federal Republic of Germany

Note added in proof. In order to avoid the difficulties with the distribution splitting in Eq. (6a) we should have defined $F$ via (Eq. (7))

$$
F(u, v):=V_{2}(u+v-1, u-v) .
$$

This leads to simple and obvious modifications of Eq.'s (8), (9), (11), (13, (28), (31), (33), (C2) and (C4). The distribution $F_{\beta}$ (Eq. (10)) has now the form [13]

$$
F_{\beta}(u, v)=u_{+}^{1-\beta} F_{\beta}(v)+u_{-}^{1-\beta} F_{\beta}(1-v)
$$

for $\beta \neq 2,3,4 \ldots$, which may be continued into the points $\beta=n=2,3,4 \ldots$ by means of locality (Eq. (32)) leading to

$$
F_{n}(u, v)=u^{1-n} F_{n}(v) \quad \text { on } \quad \mathbb{R}^{2} .
$$

Eq. (34) now reads $J_{\infty}=\left\langle F_{\beta}(u, v), g(u, v)\right\rangle$. 